\title{
Tracing few-femtosecond photodissociation dynamics on molecular oxygen with a single-color pump-probe scheme in the VUV
}

\author{
Oliver Schepp, ${ }^{1, *}$ Arne Baumann, ${ }^{1}$ Dimitrios Rompotis,,${ }^{1 \dagger}$ Thomas Gebert, ${ }^{2}$ Armin Azima,,${ }^{1,2,3}$ \\ Marek Wieland, ${ }^{1,2,3}$ and Markus Drescher ${ }^{1,2,3}$ \\ ${ }^{1}$ Institut für Experimentalphysik, Universität Hamburg, Luruper Chaussee 149, 22761 Hamburg, Germany \\ ${ }^{2}$ Center for Free-Electron Laser Science CFEL, Luruper Chaussee 149, 22761 Hamburg, Germany \\ ${ }^{3}$ The Hamburg Centre for Ultrafast Imaging CUI, Luruper Chaussee 149, 22761 Hamburg, Germany
}

(Received 17 June 2016; published 13 September 2016)

\begin{abstract}
Molecular wave-packet dynamics in oxygen are studied in the time domain, using a single-color VUVpump-VUV-probe scheme. 17-fs VUV pulses, centered at $161 \mathrm{~nm}$ are generated via high-order harmonic generation driven by an intense 800-nm pulse leading to VUV pulse energies that reach $1.1 \mu \mathrm{J}$ per pulse. An all-reflective interferometric pump-probe scheme is used for studying the delay dependence of the molecular oxygen ion signal with simultaneous nonresonant photoionization of krypton as a precise timing-reference. Access to the excited dissociative state lifetime is provided by the resulting delay-dependent $\mathrm{O}_{2}{ }^{+}$signal, ultimately limited by the molecular ionization window. The ability to use a two-photon VUV probe provides the delay-dependent detection of $\mathrm{O}^{+}$as an additional observable, extending the dissociation observation window.
\end{abstract}

DOI: 10.1103/PhysRevA.94.033411

\section{INTRODUCTION}

On the basis of its significance for many photochemical processes in nature, molecular oxygen has been the subject of several studies in the past decades. The absorption exhibited both in the ultraviolet (UV) and the vacuum ultraviolet (VUV) spectral range leads to attenuation of solar radiation and most importantly the formation of the ozone layer in the stratosphere [1]. Neutral and ionic states have been well investigated by spectroscopy (e.g, Refs. [2-4]), delay-dependent dissociative photoionization [5], and theoretical calulations (e.g, Refs. [6-9]).

The excitation of $\mathrm{O}_{2}$ in the vicinity of $160 \mathrm{~nm}$ leads to the first fully allowed transition in the so-called Schumann-Runge continuum, ultimately resulting in molecular dissociation. Still, the temporal progress of the reaction in the considered dissociation channel is the subject of an ongoing discussion [10-12]. Most studies so far have used continuous-wave VUV sources or multiphoton excitation schemes with visible laser pulses, while single-photon experiments on the relevant femtosecond time scale are rare. Farmanara et al. [10] directly observed a decay of the $\mathrm{O}_{2}{ }^{+}$signal in a 155-nm-pump, 258-nm-probe scheme and reported a time constant of $(40 \pm 20)$ fs. The quoted error originates from a probe pulse duration of $350 \mathrm{fs}$, significantly longer than the extracted time constant. Peralta Conde et al. [12], using a 160-nm-pump, 160-nm-probe scheme, concluded a time constant conceivably shorter than that which could be accessed

\footnotetext{
*oliver.schepp@desy.de

†Current address: Deutsches Elektronen Synchrotron DESY, Notkestraße 85, 22607 Hamburg, Germany.
}

Published by the American Physical Society under the terms of the Creative Commons Attribution 3.0 License. Further distribution of this work must maintain attribution to the author(s) and the published article's title, journal citation, and DOI. with the 27-fs pulses used. Regarding the earlier investigations [10] they noted that due to a longer probe wavelength different states were reached, changing the ionization detection window. In a recent study, Thrushin et al. [11] measured a decay time of 4.3 fs extracted from the $\mathrm{O}_{2}{ }^{+}$signal, upon pumping with $10-\mathrm{fs}$ pulses at $160 \mathrm{~nm}$ and probing the ionic state with multiphoton absorption at $810 \mathrm{~nm}$. The reported value was interpreted as the time until an internuclear distance on the molecular potential is reached from where more than four to five photons are needed for ionization.

In the present work an intense 5th harmonic source [13] enables sensitive multiphoton pump-probe experiments in the VUV. We report on a VUV-pump-VUV-probe experiment on molecular oxygen with intense and well-characterized sub-20-fs pulses. A Michelson-type all-reflective autocorrelation scheme prevents deterioration of the spectrotemporal properties of the VUV pulses, generated as the 5th harmonic of an 800-nm laser pulse. The single-color approach avoids strong-field ionization, which may considerably influence the molecular potentials (see, e.g, the review [14]) and even modify the reaction kinematics. While in previous studies on molecular oxygen, intrinsic constraints like the limiting ionization window prevented mapping of the complete dissociation process, we are able to extend the ionization window and follow the reaction for longer bond lengths using the $\mathrm{O}^{+}$ion as an additional observable with a one-photon pump, two-photon probe scheme.

\section{EXPERIMENTAL SETUP}

The experimental setup is shown in Fig. 1. Briefly, 5th harmonic pulses from a $13.5 \mathrm{~mJ}, 40$-fs chirped pulse amplification (CPA) Ti:sapphire laser system with a central wavelength of $800 \mathrm{~nm}$ and a repetition rate of $25 \mathrm{~Hz}$ are utilized (see Ref. [13] for a detailed description). The high-order harmonic generation setup provides sub-20-fs pulses centered at $161 \mathrm{~nm}$ and pulse energies up to $1.1 \mu \mathrm{J}$, measured with a calibrated XUV 


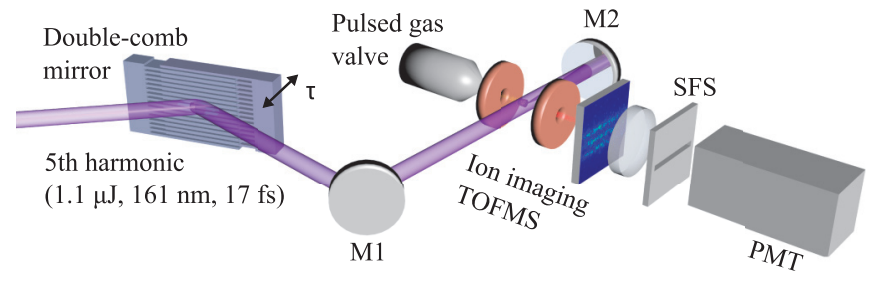

FIG. 1. Michelson-type all-reflective interferometric autocorrelator for single-color pump-probe measurements: $\tau$, optical delay; M1, dielectric multilayer mirror for $160 \mathrm{~nm}$; M2, focusing dielectric multilayer mirror; TOFMS, imaging time-of-flight mass spectrometer; SFS, spatial filtering slit for 0th diffraction order; PMT, photomultiplier tube.

photodiode. A combination of a Si reflector at Brewster's angle for $800 \mathrm{~nm}$ and dedicated dielectric stack mirrors optimized for $160 \mathrm{~nm}$ (Layertec $\mathrm{GmbH}, R_{160 \mathrm{~nm} \pm 4 \mathrm{~nm}}>0.9$ ) spectrally purify the 5 th harmonic from the fundamental and harmonic radiation. A delayed pulse pair is created using a double-comb Si mirror, acting as an all-reflective Michelson-type interferometer (details are presented in Ref. [15]), and is focused collinearly into a gas target. Ions created in the interaction region are detected with an imaging ion time-of-flight (TOF) spectrometer. A slit in front of a photomultiplier spatially filters the 0 th diffraction order created by the gratinglike structure of the double-comb $\mathrm{Si}$ mirror and allows recording the delaydependent signal for multiple-ion species simultaneously. The sampling corresponds to a delay step of 45 as.

\section{RESULTS AND DISCUSSION}

\section{A. Interferometric autocorrelation}

$\mathrm{Kr}$ gas coexpanded with the molecular $\mathrm{O}_{2}$ target serves as a precise temporal monitor for the VUV pulses. The measurement of the delay dependence of the $\mathrm{Kr}^{+}$ion signal originating from nonresonant two-photon absorption provides the second-order fringe-resolved interferometric autocorrelation (FRIAC) of the 5th harmonic pulse as shown in Fig. 2. The resulting signal $I_{\text {FRIAC }}(\tau)$ consists of three main terms depicting the intensity autocorrelation function (IAC) of the pulse and two interferometric terms, one oscillating with the fundamental pulse carrier frequency $\omega$ and a second oscillating with $2 \omega$, respectively. It is analytically given by the following expression (see details in Ref. [16]):

$$
\begin{aligned}
I_{\text {FRIAC }}(\tau)= & \text { Const }+2 \mathcal{F}_{\text {IAC }}(\tau)+2 \operatorname{Re}\left[\mathrm{F}_{1}(\tau) \exp (-i \omega \tau)\right] \\
& +\operatorname{Re}\left[\mathrm{F}_{2}(\tau) \exp (-2 i \omega \tau)\right]
\end{aligned}
$$

where $\tau$ is the optical delay and $F_{n}(\tau)$ are the composed correlation terms. The FRIAC's Fourier transform in the inset of Fig. 2 provides spectral information of the 5 th harmonic pulse and shows the expected $5 \omega_{L}(=\omega)$ and $10 \omega_{L}(=2 \omega)$ components in relation to the driving laser frequency $\omega_{L}$. A detailed analysis of the FRIAC trace, supported by a favorable signal-to-noise ratio, reveals only a minor chirp of the VUV pulses [15]. The measured trace reaches almost a contrast of $1: 8$, which is in excellent agreement with the theoretical prediction [16]. The 5th harmonic pulse duration is extracted by applying a low-pass filter that retains only the slowly

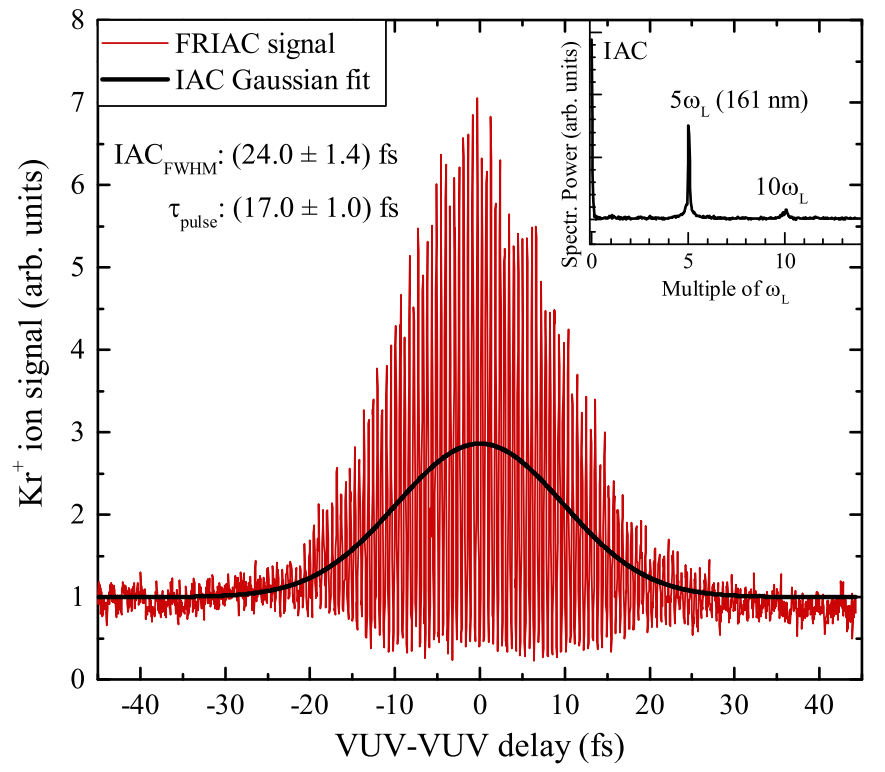

FIG. 2. Measured FRIAC utilizing nonresonant two-photon ionization of Kr. Spectral information about the pulse is given by the Fourier transform shown in the inset. The oscillating $5 \omega_{0}$ and $10 \omega_{0}$ components are visible. A pulse duration of $\tau_{p}=(17.0 \pm 1.0) \mathrm{fs}$ is obtained, assuming a Gaussian temporal profile.

varying envelope of the IAC function [Eq. (1)] and corresponds to $(17.0 \pm 1.0)$ fs. Assuming a Gaussian pulse temporal profile with a conversion factor of $\sqrt{2}$ delivers the second-order nonlinearity. This measurement, obtained in parallel with the pump-probe measurement described below, corresponds to the instrument response function, which in this case is represented by the second-order intensity autocorrelation, reflecting the experimental temporal resolution that is achieved with the current experimental setup. The accurate experimental determination of the instrument response function, paired with valid assumptions about the system's temporal response function, enables deconvolution of the intrinsic molecular dynamics time constants, when the temporal resolution is comparable or even slightly longer than the dynamics of interest.

\section{B. $\mathrm{O}_{2}$ dissociation dynamics: VUV pump-probe experiment}

The delay-dependent $\mathrm{O}_{2}{ }^{+}$ion signal generated by twophoton absorption is used as the main observable for the pumpprobe experiment as mentioned above. The photodissociation is initiated with the absorption of a VUV photon, exciting molecular oxygen approximately $0.6 \mathrm{eV}$ above the dissociation limit in the $B^{3} \Sigma_{u}^{-}$state. Upon excitation, the molecule dissociates to neutral oxygen atoms $\mathrm{O}\left({ }^{3} \mathrm{P}\right)+\mathrm{O}\left({ }^{1} \mathrm{D}\right)$ [2]. Note that predissociation from this state via coupled continuum states can be neglected as it has been shown previously by Lin et al. [17]. A delayed replica of the pump pulse provides the necessary temporal resolution for probing the dissociation dynamics by promoting a further transition to the $X^{2} \Pi_{g}$ ionic ground state of $\mathrm{O}_{2}{ }^{+}$. With this state being reached with a single photon, we are able to avoid the use of a strong multiphoton IR probe transition and its associated complications as pointed out in Refs. [11,14,18], at comparably low intensities $(\approx 3 \times$ 

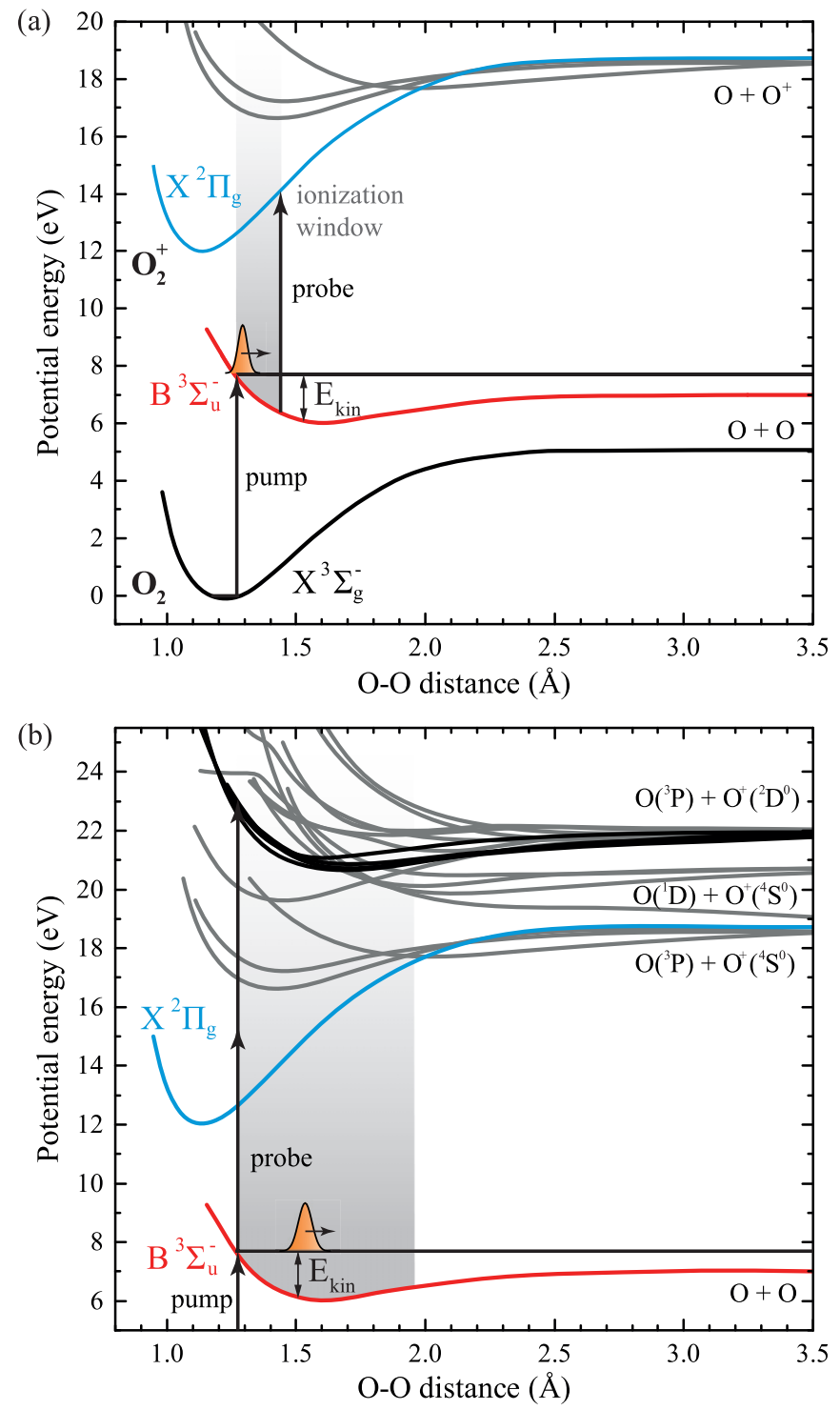

FIG. 3. Pump-probe scheme with relevant potential energy curves for $\mathrm{O}_{2}$ and $\mathrm{O}_{2}{ }^{+}$from Refs. [2,8]. (a) Time-dependent two-photon ionization via the $B^{3} \Sigma_{u}^{-}$state. The ionization window reached after a internuclear distance of $1.43 \AA$ is shown in gray. (b) Delay-dependent three-photon absorption. The utilized intense VUV pulse allows a two-photon probe transition, leading to the generation of $\mathrm{O}^{+}$ions and extending the molecular dissociation observation window.

$10^{12} \mathrm{~W} / \mathrm{cm}^{2}$ ). The delay-dependent $\mathrm{O}_{2}{ }^{+}$ion signal provides information on the wave-packet dynamics associated with the molecular dissociation. However, although dissociation is expected to be complete in some tens of femtoseconds, the observation of the molecular wave packet in the twophoton ionization scheme is limited by the temporally shorter ionization window indicated in Fig. 3(a). According to the potential energy curves used in Fig. 3(a), this time window closes at a maximum bond length of $1.43 \AA$. Beyond this distance ionization with one photon is no longer possible from the $B^{3} \Sigma_{u}^{-}$state.

Figure 4 presents the low-pass-filtered time-dependent pump-probe signal for the $\mathrm{O}_{2}{ }^{+}$ion together with the instrument

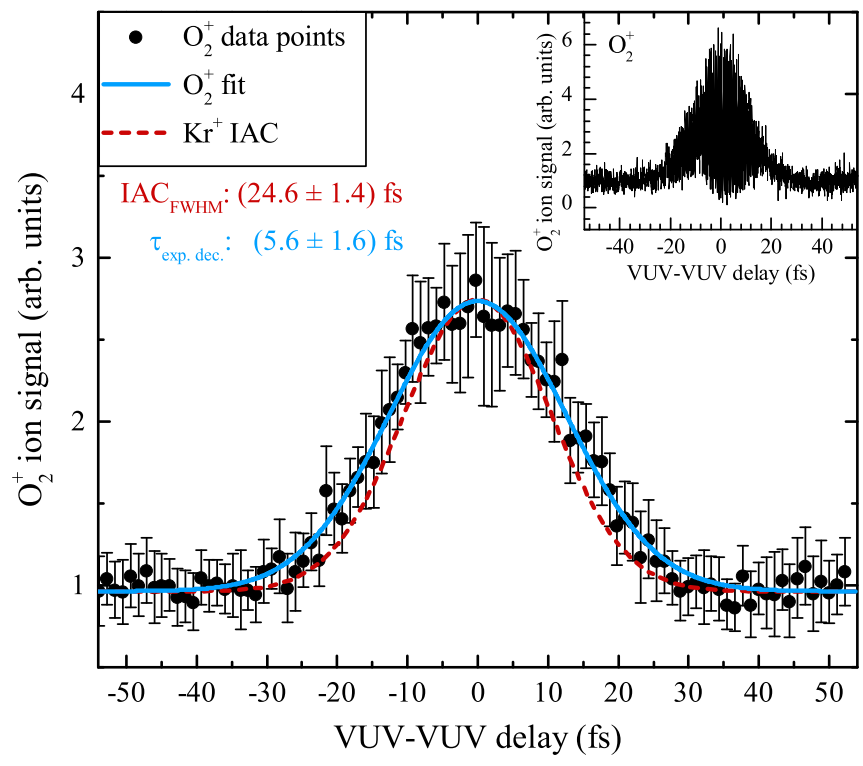

FIG. 4. Low-pass filtered $\mathrm{O}_{2}{ }^{+}$ion signal extracted from the interferometric measurement shown in the inset. A fit of the simultaneously measured IAC is shown in red and the fit including the finite ionization window for the $\mathrm{O}_{2}{ }^{+}$signal is shown in blue.

response function simultaneously measured by $\mathrm{Kr}^{+}$IAC. Modeling with a fit to a convolution between a Gaussian pulse, corresponding to the instrument response function, and a symmetric exponential decay, corresponding to the molecular response function, yields a statistically significant broadening of the $\mathrm{O}_{2}{ }^{+}$trace in comparison with the $\mathrm{Kr}^{+}$IAC. The routine has been proven in Ref. [19] and the perturbative approach is valid as long as there is no contribution of nonradiative coupled decay channels, which is valid in this case [17]. The fit of the ionic state population results in an exponential time constant of $(5.6 \pm 1.6) \mathrm{fs}$.

For the interpretation of the observed time constant we compute classical trajectories, based on energy conservation of the motion of the nuclei. The calculation, also consulted in Ref. [11], allows an estimation of the time it takes for the system to reach a specified internuclear distance. Following the same analysis as in Ref. [20] the distance as a function of time upon excitation is given by

$$
R(t)=R_{0}+\int_{0}^{t}\left(\frac{2}{\mu}\left\{E_{(t=0)}-V[R(t)]\right\}\right)^{-\frac{1}{2}} d t,
$$

where $V[R(t)]$ is the molecular state potential (extracted from Ref. [2]), $E_{(t=0)}$ the initial energy, $\mu$ the reduced mass, and $R_{0}$ the corresponding internuclear distance. It has to be emphasized that the result of these calculations can only be an estimation, since accurate knowledge of the molecular state potential and the particular pump-probe excitation scheme plays a crucial role. Solving the above Eq. (2) yields a time of 5.3 fs until the ionization window closes at an internuclear distance of $1.43 \AA$.

Experimental and calculated values are in excellent agreement. Thus, we interpret the observed decay time as the time until the nuclei reach a distance of more than $1.43 \AA$ from where the transition to the $X^{2} \Pi_{g}$ state is no longer 

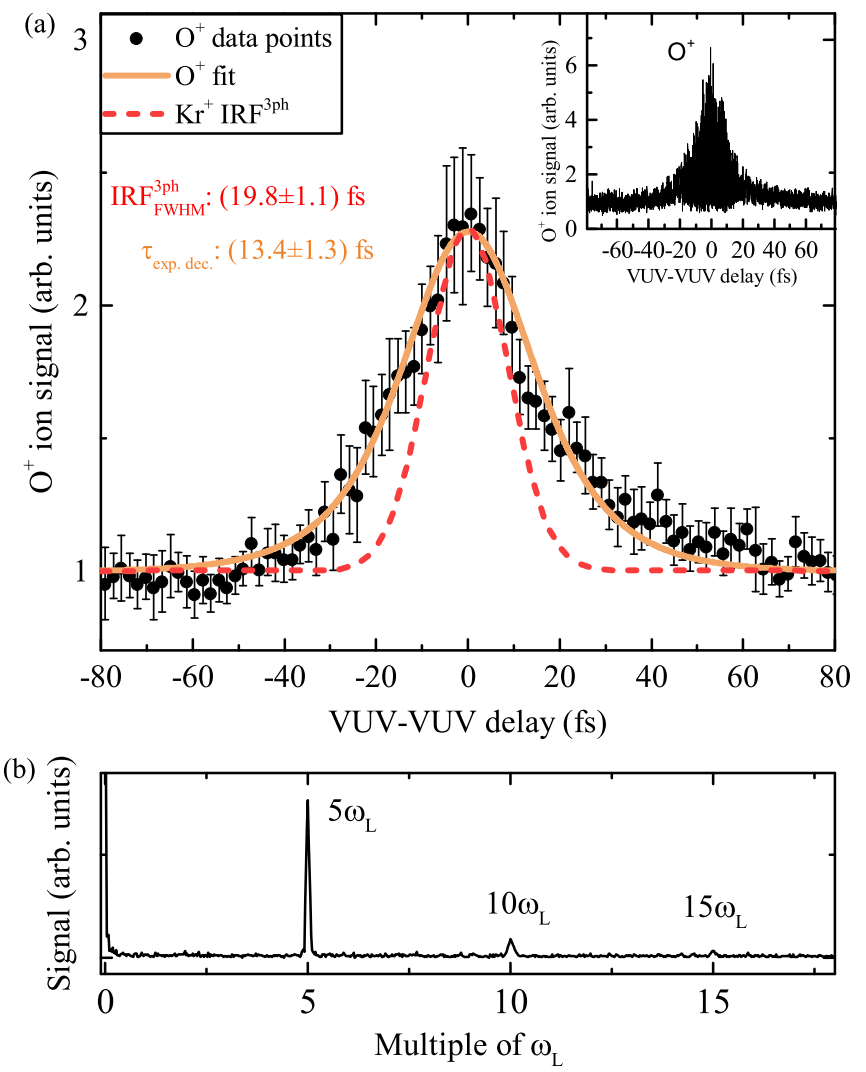

FIG. 5. (a) Low-pass filtered $\mathrm{O}^{+}$ion signal extracted from the interferometric measurement shown in the inset. The third-order instrument response function is shown in red and the decay fit of the $\mathrm{O}^{+}$signal is shown in yellow. (b) $\mathrm{O}^{+}$frequency-domain pumpprobe ion signal obtained via Fourier transform of a high-resolution interferometric scan.

accessible with a single $7.7-\mathrm{eV}$ photon and the ionization probability drops dramatically. This observation is compatible with the results presented in Ref. [12] where no pump-probe signal broadening has been observed owing to a longer VUV pulse. Our results are also in agreement with those in Ref. [11], where a slightly shorter time constant was measured, due to a different probing scheme with strong-field multiphoton ionization in the infrared. By following a weakfield approach we experimentally determined the duration of the ionization window associated with a photon energy of $7.7 \mathrm{eV}$.

The available energetic VUV pulses allow the generation of $\mathrm{O}^{+}$ions after three-photon absorption. Taking advantage of this fact we additionally utilize a multiphoton pump-probe scheme by recording the delay dependence of the $\mathrm{O}^{+}$ion signal. Figure 5(a) shows the recorded $\mathrm{O}^{+}$ion signal exhibiting a distinct broadening in comparison with the third-order instrument response function, deduced from the experimentally determined $\mathrm{Kr}^{+}$IAC. The peak-to-background ratio is less than the theoretically expected $1: 32$ and $1: 10$ for the interferometric and the low-pass-filtered trace, respectively, which is here mainly attributed to imperfectly balanced interferometer branches and a lower signal-to-noise ratio obtained with the $\mathrm{O}^{+}$ ion signal. The Fourier transform of the interferometric $\mathrm{O}^{+}$ion signal scanned with high resolution [Fig. 5(b)] reveals a $15 \omega_{L}$ frequency component in line with a three-photon $\left(3 \times 5 \omega_{L}\right)$ excitation scheme [16]. The absence of further spectral components also excludes the participation of harmonics besides the 5 th order. $\mathrm{O}^{+}$ions can result after two-photon absorption in the $B^{3} \Sigma_{u}^{-}$molecular oxygen state $(1+2$ photons $)$ or after one-photon absorption in the $X^{2} \Pi_{g}$ state $(2+1$ photons). The latter process would probe a molecular wave packet in the bound $X^{2} \Pi_{g}$ state and would, consequently, create a temporal revival pattern in the signal when the wave packet leaves the ionization window at short internuclear distances. The absence of such periodically appearing features in the observed delay dependence rather identifies a two-photon absorption in the $B^{3} \Sigma_{u}^{-}$molecular oxygen state as the origin of the $\mathrm{O}^{+}$signal.

Following the same analysis as in the previous paragraph an exponential decay with a time constant of (13.4 \pm 1.8$)$ fs can be deconvoluted using the third-order instrument response function. Using again Eq. (2), the experimentally determined time constant corresponds to an internuclear distance of $1.95 \AA$, according to the same classical trajectory calculation as described above. The energy region accessed by the two-photon probe contains a large number of bound and repulsive states, which converge to the three different dissociation limits shown in Fig. 3(b), considering the selection rules for a two-photon absorption process starting from the excited B ${ }^{3} \Sigma_{u}^{-}$molecular state. The excitation is resonant with four of them for zero delay and the delay-dependent signal indicates that the ionization probability is mainly influenced by these higher excited ionic states. This additional observable, enabled by a multiphoton VUV probe transition, allows us to follow the dissociation dynamics for a considerably longer bond length as compared to previous studies [10-12]. The present results are in accordance with classical trajectory simulations, while excluding an ultrafast dissociation process as suggested by using a single VUV photon probe. In addition we experimentally verify that the choice of an appropriate probe transition is necessary in order to be able to follow the molecular dynamics, while avoiding perturbations by the measuring scheme.

\section{CONCLUSION}

The photodissociation dynamics of molecular oxygen in the $B^{3} \Sigma_{u}^{-}$state have been studied by recording the delaydependent $\mathrm{O}_{2}{ }^{+}$and $\mathrm{O}^{+}$ion yield with a two- and three-photon VUV interferometric pump-probe scheme. A VUV-pumpVUV-probe experiment utilizing a short, energetic pulse at $161 \mathrm{~nm}$ yields a delay-dependent $\mathrm{O}_{2}{ }^{+}$ion signal resulting in an extracted time constant of $(5.6 \pm 1.6)$ fs, modeled using a symmetric exponential decay function. This value is in excellent agreement with classical trajectory calculations. The availability of energetic sub-20-fs VUV pulses enables us to extend multiphoton time-resolved spectroscopy in the VUV, utilizing a two-photon VUV probe that does not influence the system under study, contrary to pump-probe schemes using a multiphoton IR probe. The VUV pulses used in this experiment are sufficiently intense to alternatively permit a two-photon VUV-probe transition. In this case the time dependence of the $\mathrm{O}^{+}$ions generated by the two-photon VUV probe can 
be used to extend the molecular dissociation observation. The observed transient with a exponential time constant of $(13.4 \pm 1.8)$ fs indicates that the time-dependent ionization probability relevant for the interpretation of the pump-probe experiment is mainly influenced by the final excited ionic states.

\section{ACKNOWLEDGMENTS}

We thank the German Science Foundation within the SFB 925 (Light Induced Dynamics and Control of Correlated Quantum Systems), Subproject A2, for financial support as well as the GRK 1355 (Physics with New Coherent Light Sources) and the Hamburg Centre for Ultrafast Imaging (CUI).
[1] D. H. Parker, Acc. Chem. Res. 33, 563 (2000).

[2] P. Krupenie, J. Phys. Chem. Ref. Data 1, 423 (1972).

[3] K. P. Huber and G. Herzberg, Molecular Spectra and Molecular Structure (Van Nostrand Reinhold, New York, 1979).

[4] T. Tanaka, H. Yoshii, Y. Morioka, T. Hayaishi, K. Ito, and R. I. Hall, J. Chem. Phys. 108, 6240 (1998).

[5] P. Cörlin, A. Fischer, M. Schönwald, A. Sperl, T. Mizuno, U. Thumm, T. Pfeifer, and R. Moshammer, Phys. Rev. A 91, 043415 (2015).

[6] N. H. Beebe, E. W. Thulstrup, and A. Andersen, J. Chem. Phys. 64, 2080 (1976).

[7] R. P. Saxon and B. Liu, J. Chem. Phys. 67, 5432 (1977).

[8] C. M. Marian, R. Marian, S. D. Peyerimhoff, B. A. Hess, R. J. Buenker, and G. Seger, Mol. Phys. 46, 779 (1982).

[9] K. Takeshita, Y. Sadamatu, and K. Tanaka, J. Chem. Phys. 122, 044302 (2005).

[10] P. Farmanara, O. Steinkellner, M. T. Wick, M. Wittmann, G. Korn, V. Stert, and W. Radloff, J. Chem. Phys. 111, 6264 (1999).
[11] S. A. Trushin, W. E. Schmid, and W. Fu, J. Phys. B 44, 165602 (2011).

[12] A. Peralta Conde, J. Kruse, O. Faucher, P. Tzallas, E. P. Benis, and D. Charalambidis, Phys. Rev. A 79, 061405 (2009).

[13] D. Rompotis, T. Gebert, M. Wieland, F. Karimi, and M. Drescher, Opt. Lett. 40, 1675 (2015).

[14] J. H. Posthumus, Rep. Prog. Phys. 67, 623 (2004).

[15] T. Gebert, D. Rompotis, M. Wieland, F. Karimi, A. Azima, and M. Drescher, New J. Phys. 16, 073047 (2014).

[16] J.-C. Diels and W. Rudolph, Ultrashort Laser Pulse Phenomena: Fundamentals, Techniques, and Applications on a Femtosecond Time Scale (Academic Press/Elsevier, Amsterdam, 2006).

[17] J. J. Lin, D. W. Hwang, Y. T. Lee, and X. Yang, J. Chem. Phys. 109, 1758 (1998).

[18] A. González-Castrillo, A. Palacios, H. Bachau, and F. Martín, Phys. Rev. Lett. 108, 063009 (2012).

[19] S. Pedersen and A. H. Zewail, Mol. Phys. 89, 1455 (1996).

[20] A. H. Zewail, Faraday Discuss. Chem. Soc. 91, 207 (1991). 